Int. J. Dev. Biol. 58: 513-520 (2014)

doi: $10.1387 / \mathrm{ijdb} .140087 \mathrm{rk}$

\title{
Spiral cleavages determine the left-right body plan by regulating Nodal pathway in monomorphic gastropods, Physa acuta
}

\author{
MASANORI ABE, HIROMI TAKAHASHI and REIKO KURODA* \\ Research Institute for Science and Technology, Tokyo University of Science, Yamazaki, Noda-shi, Chiba, Japan
}

\begin{abstract}
The handedness of gastropods is genetically determined, but the molecular nature of the gene responsible and the associated mechanisms remain unknown. In order to characterize the chiromorphogenesis pathway starting from the gene to the left-right asymmetric body plan, we have closely analyzed the cytoskeletal dynamics of the Physa (P.) acuta embryo, a fresh water non-dimorphic sinistral snail, during the early developmental stage by mechanically altering the handedness of the embryos at the critical spiral third cleavage. A fertile situs inversus was created and the nodal-Pitx gene expression patterns were completely mirror imaged to the wild type at the trochophore stage. Together with our previous work on Lymnaea (L.) stagnalis, we could show that chirality is established at the third cleavage, as dictated by the single handedness-determining gene locus, and then chirality information is transferred via subsequent spiral fourth and fifth cleavages to the later developmental stage, dictating the nodal-Pitx expression pathway. The cytoskeletal dynamics of manipulated and non-manipulated embryos of sinistral $P$. acuta and dextral dominant L. stagnalis are compared.
\end{abstract}

KEY WORDS: spiral cleavage, nodal pathway, chirality, shell coiling, gastropod

\section{Introduction}

Left and right handedness of animals is displayed internally and in some species externally as well, and they are genetically programmed throughout the developmental stages. Several different mechanisms for I-r (left-right) asymmetry determination have been proposed for vertebrates and invertebrates (Spéder et al., 2007; Vandenberg and Levin, 2009). For examples, in vertebrates, I-r asymmetry is determined by directional nodal flow across the embryonic midline (Nonaka et al., 1998; Nonaka et al., 2002; Blum et al., 2009), whereas in invertebrates such as Drosophila and $C$. elegans, the actin cytoskeleton and an associated type I myosin, and G-alpha protein regulating spindle orientation seem to be involved, respectively (Hozumi et al., 2006; Spéder et al., 2006; Bergmann et al., 2003). Levin suggested some general mechanisms involving gap junctions and $\mathrm{H}^{+} / \mathrm{K}^{+}$-ATPase activity as the initial symmetry-breaking steps (Levin et al., 2002; Adams et al., 2002; Vandenberg and Levin, 2009). In contrast, nodal-Pitx gene expression cascades are well conserved among animal phylum and class including vertebrates, ascidians and Echinoidea, and is known to play an important role in the handedness determination
(Boorman and Shimeld, 2002; Duboc et al., 2008). Recently, nodal and Pitx genes are found to operate in gastropods as well (Grande and Patel, 2009a; Kuroda et al., 2009; Grande and Patel, 2009b).

We have been working on the mechanisms of body-handedness determination by using fresh water snail $L$. stagnalis, because it has unique characteristics ideal for the developmental study of left and right handedness determination, not only for gastropoda but for other animal species including vertebrates as well. The genus Lymnaea is dimorphic, that is, both the dextral and sinistral strains exist in the wild (Hosoiri et al., 2003). The dominant dextral and recessive sinistral snails possess a mirror-image body of each other including the shell coiling and the internal organs. The chirality is hereditary, determined by a single locus that functions maternally which was revealed genetically by ourselves (Kuroda et al., 2009, Kuroda, 2014) and also by DNA mapping using RAD-Seq and Fibre-FISH techniques (Liu et al., 2013).

Gastropods undergo characteristic spiral cleavage during the

Abbreviations used in this paper: Pitx, pituitary homeobox; TGF- $\beta$, transforming growth factor- $\beta$.

\footnotetext{
*Address correspondence to: Reiko Kuroda. Research Institute for Science andTechnology, Tokyo University of Science, 2641 Yamazaki, Noda-shi, Chiba 278-8510 Japan.E-mail: rkuroda@rs.tus.ac.jp
}

Accepted: 20 June 2014.

ISSN: Online 1696-3547, Print 0214-6282 
Control Physa embryo
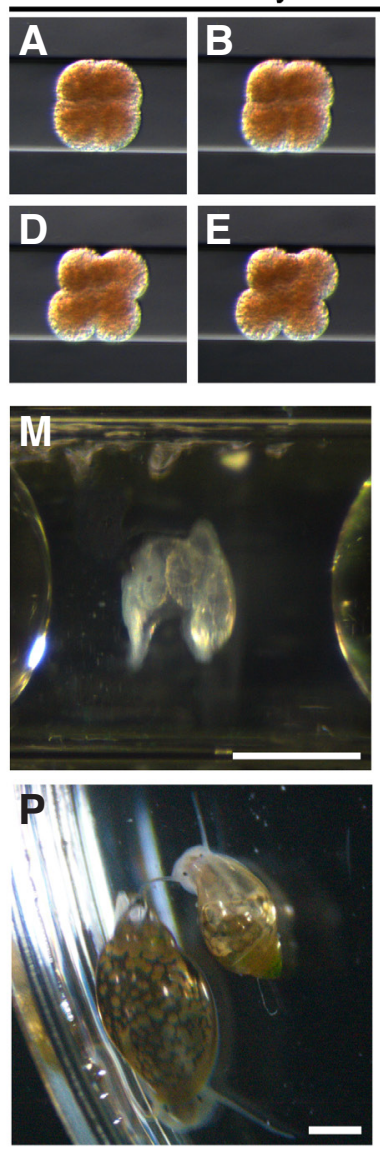

Reversed Physa embryo
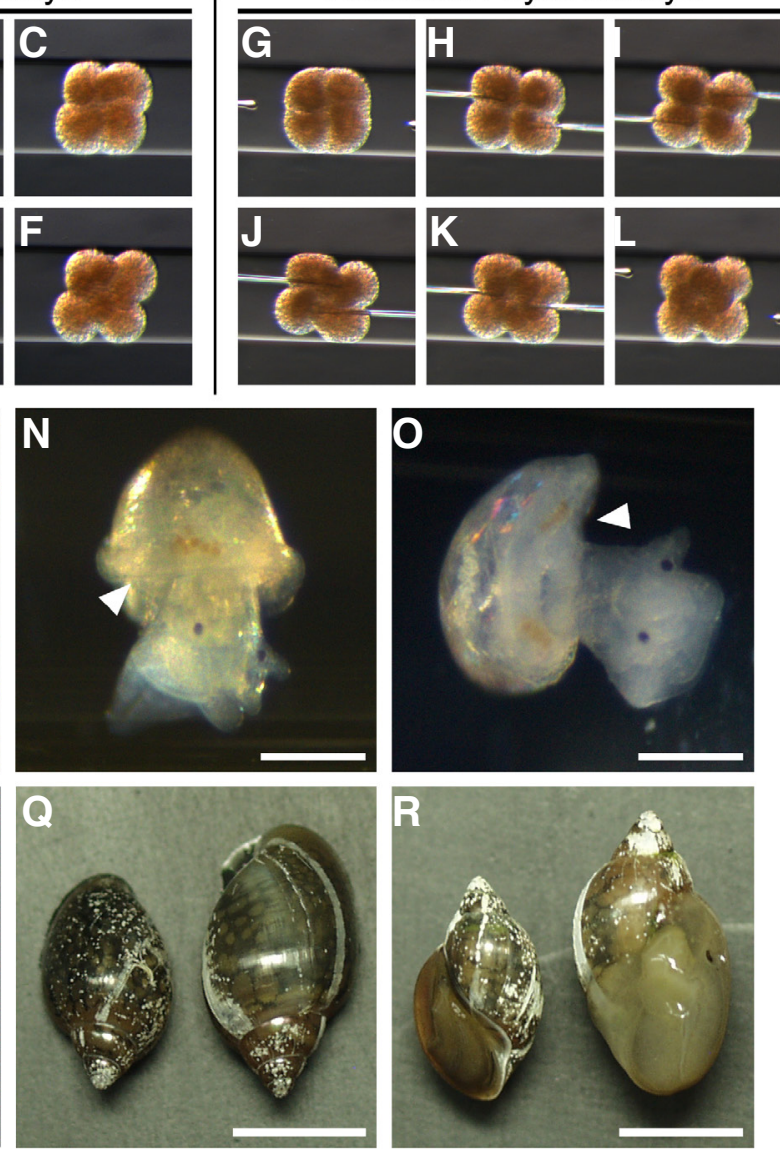
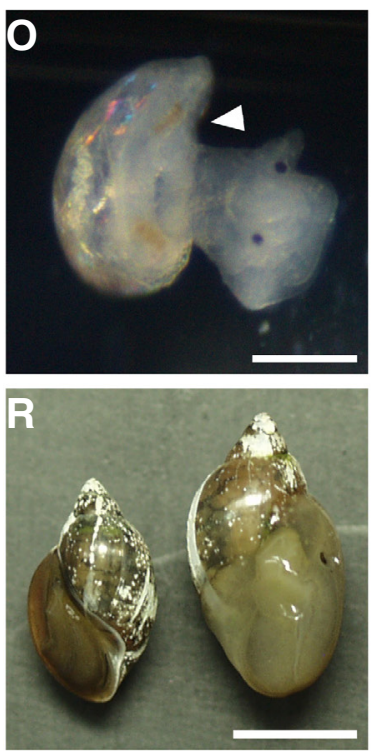

Fig. 1. Creation of reversed Physa acuta. At the third cleavage (4-8 cell stage), (A-F) micromeres rotation is sinistral-type anti-clockwise (control). (G-L) The rotation was forced to clockwise direction by pushing the micromeres gently with two glass rods until the new cell contacts were formed. (M-O) After the manipulation, the embryos were cultured in a glass capillary filled with capsule fluid. (M) The reversed embryo developed to veliger stage. The reversed (N) and control (0) embryos developed to juvenile stage, ready for out-of-capillary cultivation. White arrowheads indicate the aperture side. (P-R) Comparison of the shell coiling between the normal snail developed in a capsule after hatching, and the reversed one cultured in a capillary after taking out of the capillary. The control (left) and reversed (right) snails were photographed together side by side at the stage of $37(\mathbf{P}), 83(\mathbf{Q})$ and $93(\mathbf{R})$ days after taking out the reversed snail from the capillary. Scale bars: (M), $0.5 \mathrm{~mm}$; $(\mathrm{N}, O) 0.2 \mathrm{~mm}$; $(P) 1 \mathrm{~mm}$; $(Q, R) 5 \mathrm{~mm}$.

third to fifth cleavages (Morrill, 1982; Verdonk and van den Biggelaar, 1983; Meshcheryakov, 1990). The handedness of snails can be distinguished easily at the third cleavage (i.e., 4 to 8 cell stage) as a quartet of micromeres is rotated with respect to that of sister macromeres in a clockwise sense in a dextral embryo, while in an anticlockwise manner in a sinistral embryo, looking from the animal pole. The rotation direction of protruding micromeres is always referred as looking down from the animal pole. It had been believed that all the developmental steps for the sinistral and dextral embryos are mirror images of each other based on conventional microscopic observations (Crampton, 1894; Meshcheryakov and Beloussov, 1975). However, by close analysis of the temporal and spatial behaviour of cytoskeletons of the dextral and the sinistral $L$. stagnalis during the early cleavages, we have revealed non-mirrorimage cytoskeletal dynamics at the third cleavage (Shibazaki et al., 2004). A helical deformation of blastomere shape (spiral deforma-

tion, SD) was observed at the animal hemisphere in metaphase to anaphase, together with helical spindle orientation (spindle inclination, $\mathrm{SI}$ ) in the case of dominant dextral embryos. In contrast, no SD and SI were observed for the recessive sinistral animals. Thus, the blastomere shape and the spindle orientation as well as the timing of helicity generation are different for the dextral and sinistral embryos within a species, and are far from enantiomorphs. By using congenic strains we had generated, we could further show that the dextral-specific cytoskeletal dynamics (SD and the consequent $\mathrm{SI}$ ) is genetically strongly linked to the single handedness-determining gene (Shibazaki et al., 2004; Kuroda et al., 2009, Kuroda, 2014).

More recently, we succeeded in creating healthy and fertile creatures with body handedness reversed to what is inherited, by blastomere micromanipulation at the third cleavage stage. We have shown that the asymmetric Nodal pathway does operate for the determination of the asymmetric body plan in $L$. stagnalis as well. When chirality at the 8-cell embryos was reversed by micromanipulation, the nodal and Pitx expression sites were completely shifted to the mirror-image positions (Kuroda et al., 2009).

One may argue that the success in reversing genetically determined handedness by mechanical manipulation is due to the fact that $L$. stagnalis is dimorphic. This means that the species may somehow have a potential, by nature, to accommodate the reversing physical force. Further, it is interesting to see whether similar handednessdetermining mechanisms for $L$. stagnalis operate in sinistral-dominant snails as well. Thus, in this study, we tried to create dextral snails of the genus Physa, a sinistral gastropod, whose dextral strains have not been found. We followed and compared the cytoskeletal dynamics at the third and fourth spiral cleavages in detail for the mechanically reversed and non-manipulated embryos. We cloned the $P$. acuta orthologues of nodal and Pitx genes and investigated the expression patterns by whole mount in situ hybridization at the late trochophore stage. We could create a mirror image animal whose strain does not seem to exist in the wild, and showed that the third cleavage determines the following I-r asymmetric development, passing chirality information to the fourth and fifth spiral cleavages and then to the Nodal-Pitx pathway, similar to $L$. stagnalis, but with opposite chirality. The implications of these results are discussed in perspective of spiralian and vertebrates I-r asymmetry determination.

\section{Results}

\section{Creating a reversed $P$. acuta individual by mechanically chang- ing the 8-cell blastomere arrangement}

Micromanipulation on cleavage directions was performed using the methods we have developed previously for L. stagnalis (Kuroda 

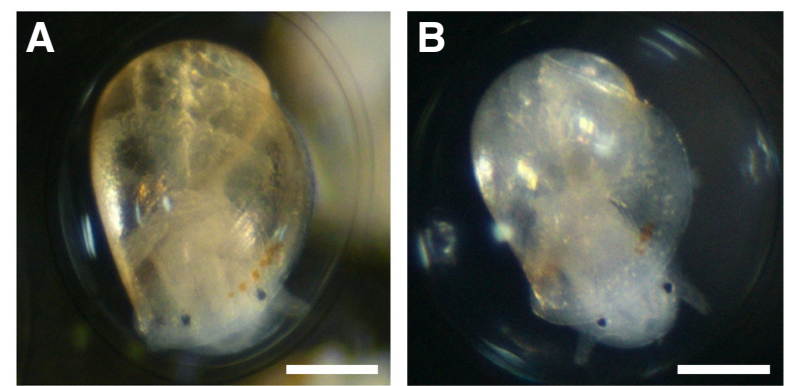

Fig. 2. The offspring chirality. Juvenile snails of $\mathrm{P}$. acuta in a capsule. (A) Oviposited by normal and (B) by the chirality-reversed mother. Scale bar: $0.2 \mathrm{~mm}$.

et al., 2009). Wild P. acuta undergoes anticlockwise rotation at the third cleavage (Fig. 1 A-F). SD was observed as in dextral L. stagnalis, though in opposite direction. We have previously reported that the mirror image relationship holds in terms of blastomere shape and spindle orientation, for the dominant sinistral and dominant dextral snails across species (Shibazaki et al., 2004). Thus, as in the case of $L$. stagnalis, micromanipulation was applied from the metaphase $\sim$ anaphase stage. A constant mechanical force was applied to the protruding micromeres at the animal pole of each blastomere in the directions opposite to the normal third cleavage (Fig. $1 \mathrm{H}-\mathrm{K}$ ) until contacts between newly formed micromeres and macromeres were established (Fig. 1L).

Judging from the blastomere configurations and intercellular contacts, $62 \%(92 / 149)$ of sinistral embryos were successfully reversed to "dextralized" 8-cell stage embryos. We incubated the manipulated embryos up to 33-64 cell stage and transferred only the healthy looking embryos with proper compaction, into glass capillary tubes filled with the natural capsular fluid (70/92). Seven out of the seventy embryos developed into a veliger stage (Fig. 1M) and six of them developed to juvenile snails with completely reversed features (Fig. $1 \mathrm{~N}$ ). This can be compared with control juveniles without any manipulation and grown inside the capsules (Fig. 1O). It is clearly seen that the aperture is on the right-hand side of the body and the shell coils in the right-handed way for the reversed embryos (Fig. 1N), while in the left-hand side of the body and left-handed shell coiling for the control (Fig. 10). The six manipulated juvenile snails were taken out of the

Fig. 3. The fourth cleavage of chirality-reversed embryos. (A-J) Time-lapse images of the fourth cleavage of manipulated (Rev) and non-manipulated (Con) embryos. Pairs of Rev (top) and Con (bottom) embryos at the similar development stage were placed side by side in a similar orientation and shot in a frame during the course of the fourth cleavage. Time was conveniently counted from the end of compaction after the third cleavage, and indicated at each embryonic stage. Development of Rev was slightly advanced than Con and time offset was 4 min 30 sec. Black arrows indicate spiral cleavage directions. Scale bar: $100 \mu \mathrm{m}$. Heads of arrows are pointed towards the animal pole.
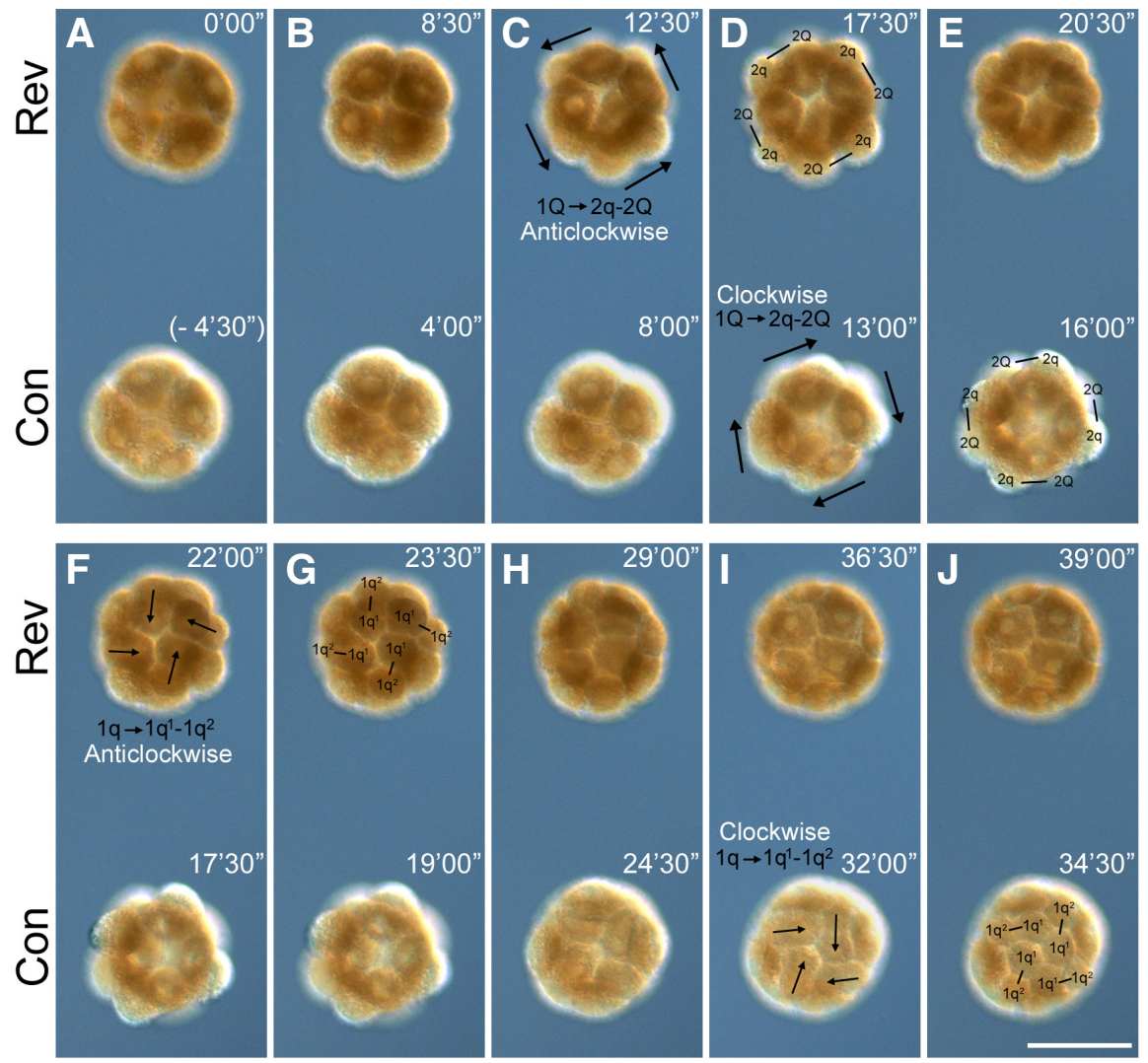

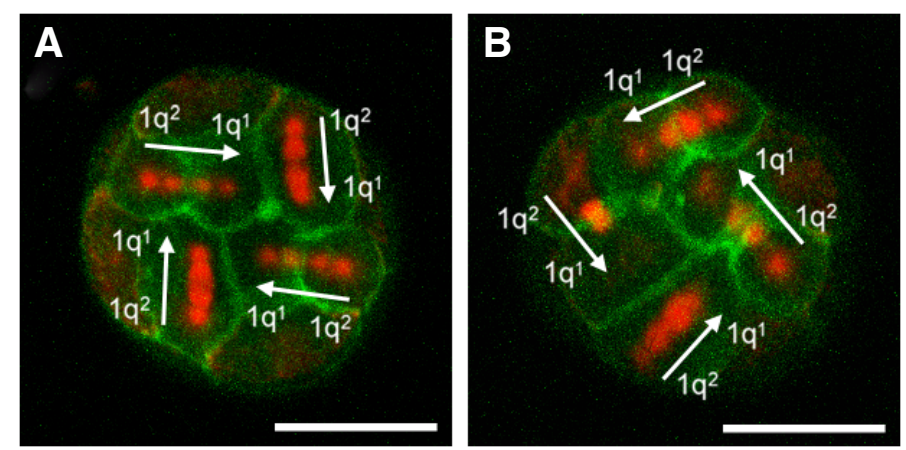

Fig. 4. Spindle orientation at the fourth cleavage of wild and reversed embryos. Division of first quartet micromeres in the fourth cleavage of control (A) and reversed (B) embryos. Animal views (z-stack, 16 sections at every $2.2 \mu \mathrm{m})$. Fixed embryos were stained for actin filament (green) and $\beta$-tubulin (red). White arrows indicate the orientation of the spindles. Scale bar: $50 \mu \mathrm{m}$. Heads of arrows are pointed towards the animal pole.

easy to place the control and reversed embryos side by side in the same orientation and at similar developmental stages, but these figures conclusively show the mirror-image relationship in cleavage patterns and different developmental behaviour in time course. Fig. 3 shows representative such images. Time for the fourth cleavage was conveniently set to 0 , when the blastomere compaction after the third cleavage was about to end. The developmental stage for the control (Con) and reversed (Rev) embryos in a frame is a little offset, with slightly more advanced cleavage stage for Rev (4'30" faster as compared with Con).

In the control embryos, first quartet macromeres (1Q) underwent spiral cleavage in a clockwise manner ( $t=13^{\prime} 00^{\prime \prime}$, Fig. 3D Con) to form second quartet micromeres (2q, $t=16$ '00', Fig. 3E Con). At $\mathrm{t}=24^{\prime} 30$ " (Fig. 3H Con), compaction after the first macromere cleavage started, and still in the process of compaction, first quartet micromeres (1q) went through spiral cleavage dextrotropically $(t=$ 32 '00", Fig. 3 I Con) to produce $1 q^{1}$ and $1 q^{2}$.

Similar sequential cleavages of $1 Q$ and $1 q$ occurred for the reversed embryos ( $t=12$ '30", Fig. $3 \mathrm{C}$ Rev, and $t=22$ '00", Fig. 3F Rev, respectively), but the rotation was in an anticlockwise sense in both cases. The compaction after the macromere cleavage was not obvious, and the second micromere cleavage started sooner. Thus, the time duration between the $1 \mathrm{Q}$ and $1 \mathrm{q}$ divisions in the fourth cleavage was much shorter for the reversed embryos ( $\mathrm{n}$ $=3)$ as compared with the control $(n=9)$. There seems to be a slight difference in the cytoskeletal behavior of the fourth cleavage between the reversed and control embryos. However, both kept the features of alternating rotation direction in the successive third and fourth cleavages, and of the non-synchronous division of $1 Q$ and $1 \mathrm{q}$ in the fourth cleavage.

Microfilaments and mitotic spindles at the fourth cleavage were visualized by double staining of filamentous actin and microtubule by fluorescently labeled phalloidin (green) and anti- $\beta$-tubulin antibody (red), respectively (Fig. 4). At the stage of 1q cleavages corresponding to Fig. 3F Rev and Fig. 3I Con, spindles are oriented in an opposite sense: clockwise for the control (Fig. 4A) and anticlockwise for the mechanically reversed (Fig. 4B) embryos. Thus, we can say that the spindle orientation at the fourth spiral cleavage stage is controlled by spatial arrangement of blastomeres at the previous spiral cleavage, i.e., the third cleavage.

\section{Asymmetric nodal-Pitx expression system is conserved in Physa embryogenesis and the expression patterns are regu- lated by the spiral cleavage direction at the 8 cell stage}

To visualize the expression patterns of nodal and Pitx genes in $P$. acuta, we cloned the orthologues and determined the amino

A
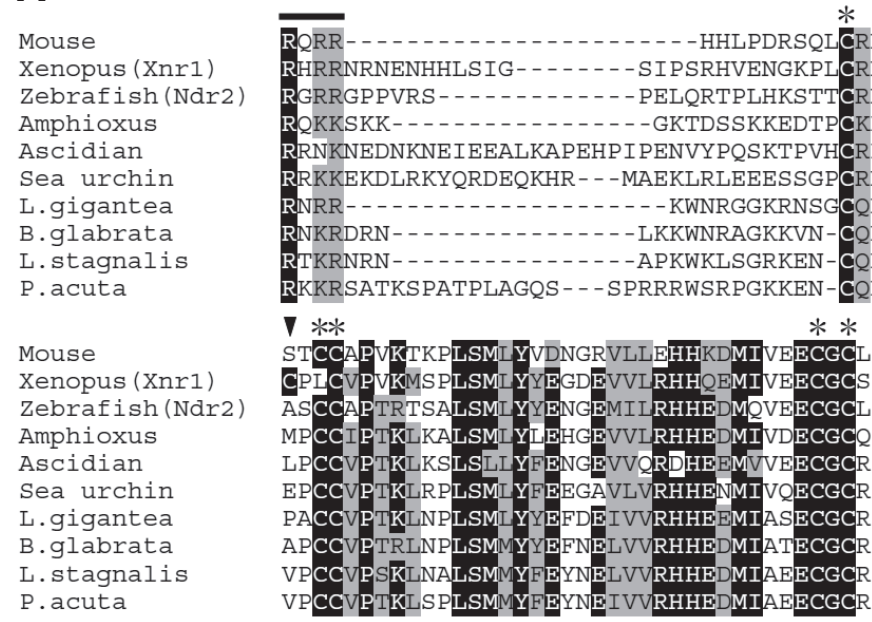

\section{B}

Mouse (Pitx2)
Amphioxus
Ascidian
Sea urchin
L.gigantea
B.glabrata
L.stagnalis
P.acuta

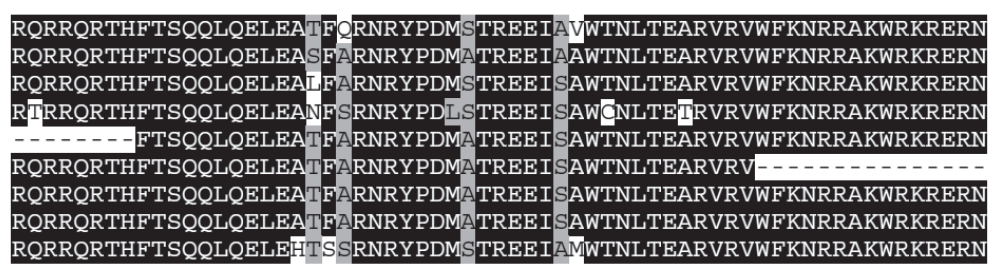

Fig. 5. Amino acid alignment. (A) Comparison of the mature domain of P. acuta Nodal (PaNodal) and other nodal-related proteins in other animals. Sequence ends are arranged at the putative cleavage site (Bold bar). The seven cysteins conserved in the mature domain are indicated by asterisks. One conserved cysteine position is shifted in Xenopus, and it is indicated by triangle. (B) Comparison of the homeobox domain of P. acuta Pitx (PaPitx) and otherPitx-related proteins. Black and grey backgrounds indicate identical and similar residues, respectively. 

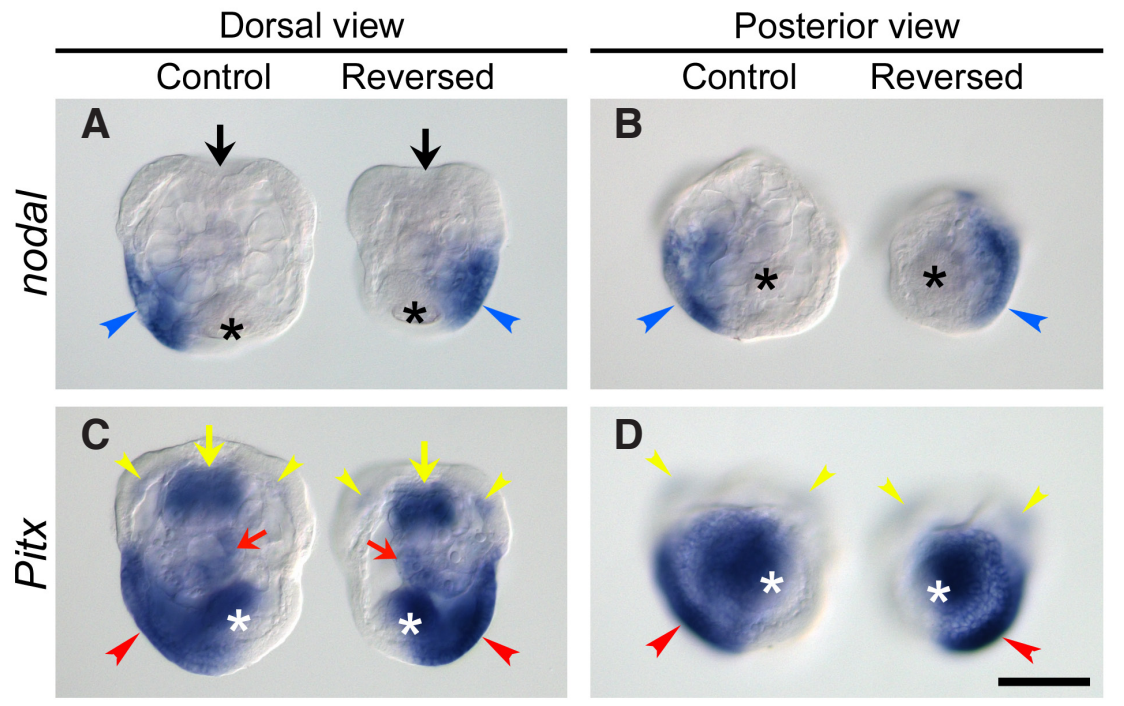

Fig. 6. nodal and Pitx expression in control and reversed $\boldsymbol{P}$ acuta embryos. Pairs of control and reversed embryos were placed side-by-side and pictured in a same frame. (A,C) Dorsal views; $(B, D)$ posterior views. $(\mathbf{A}, \mathbf{B})$ nodal is expressed in the left side posterior-lateral ectoderm (blue arrowhead) near the shell gland (marked by an asterisk) for the control, while in the right side posterior-lateral ectoderm near the shell gland for the reversed. (C,D) Pitx is expressed clearly asymmetrically in the left or right side of posterolateral ectoderm covering a part of shell gland for the control and reversed embryos, respectively (red arrowhead), and less asymmetrically in stomodeum (yellow arrow), visceral mass (red arrow), and very weakly in cephalic plates (yellow arrowhead). Scale bar: 100um.

acids sequences. All members of the nodal subfamily of the TGF- $\beta$ superfamily are synthesized as large precursor molecules, which are cleaved to release a mature domain (Kingsley, 1994; Hogan, 1994). Fig. 5A shows the amino acid sequence alignment of the mature domain of nodal orthlogue of $P$. acuta ( $P a N o d a l)$ with that of other organisms including $L$. stagnalis, Biomphalaria glabrata and Lottia gigantea. The domains are highly conserved including the cysteine residues (indicated with asterisk in Fig. 5A) among them. Pitx orthlogue gene of $P$. acuta ( $P a P i t x)$ contains the homeobox domain which is also highly conserved among many animals spanning from vertebrates to $D$. melanogaster and Gastropoda as shown in Fig. 5B. Thus, PaNodal and PaPitx are expected to function in a similar manner at least to other gastropods (Grande and Patel, 2009a).

Using the PaNodal and PaPitx sequences, whole mount in situ hybridizations were carried out at the late trochophore stage for the manually left-right inverted and non-manipulated embryos. For the non-manipulated sinistral $P$. acuta, nodal was expressed in the left lateral ectoderm (indicated with blue arrow head) near the left side of the shell gland (asterisk) (Fig. 6A, B Control). Pitx expression was detected clearly asymmetrically in the ectodermal lateral-posterior region (red arrowhead) partially covering the shell gland on the left side of the trochophore larva snails. It was also expressed in the stomodeum (yellow arrow), visceral mass (red arrow) and weakly in cephalic plates (yellow arrow head) less asymmetrically (Fig. 6 C, D Control). The same expression patterns were observed in embryos grown inside the capsules, but no signal was detected using sense probes (data not shown). When chirality at the 8-cell stage was reversed by micromanipulation, the nodal and Pitx expression patterns at the late trochophore stage were completely reversed as is clearly seen in Fig. 6A, B Reversed and Fig. 6 C, D Reversed, respectively. The results are similar to the case observed in $L$. stagnalis. Thus, in $P$. acuta as well, the blastomere arrangement at the 8-cell stage dictates the zygotic Nodal signaling pathway.

\section{Discussion}

\section{The third cleavage determines the organismal handedness}

Chirality inversion at the 8-cell stage embryos resulted in situs inversus in $P$. acuta. Unlike $L$. stagnalis, $P$. acuta is not dimorphic and does not seem to adopt a series of opposite-sense spiral-rotation, i.e., anti-clockwise, clockwise and anti-clockwise rotation for the third, fourth and fifth cleavages, respectively, in the wild (Schilthuizen and Davison, 2005). Although dextral shells/individuals were reported as a very rare collectors' item in 19th and early 20th centuries (Williams, 1887; Pelseneer, 1920), these might have been a result of malformation and not of genetic changes. No dextral strains of $P$. acuta have been reported. Even so, cytoskeletal dynamics was not upset by the forced dextrality at the third cleavage and performed the almost normal subsequent spiral cleavages. Thus, the creation of mirror-image individuals is not restricted to species which exhibit dimorphism in the wild, and it may be workable in other members of spiralia.

Spiral cleavage where the cleavage planes are at oblique angles to the animal-vegetal axis of the egg occurs during the second (or may be even the first?) to fifth cleavages in alternating rotation direction, but they are most notable during the third to fifth cleavages (Meshcheryakov, 1975; Verdonk and van den Biggelaar, 1983; Lambert, 2010). Embryos manually forced to rotate clockwise way at the third cleavage opposite to the wild type, underwent anticlockwise rotation first during the macromere division and then during the micromere division as well, in the subsequent fourth cleavage. We only pushed the forming micromeres (1q), during the third cleavage to create mirror-image 8-cell embryos, and did not touch the vegetal hemisphere of macromeres $(Q=A, B, C$ and $D)$. In the subsequent fourth cleavage, both $1 Q$ and $1 q(q=a, b, c$ and d) rotated opposite sense to the third cleavage, indicating it is not the result of intra-cellular event caused by cell pushing but of the relative placement of $1 Q$ and $1 q$, i.e. micromere - macromere contact that determines the direction of the next spiral cleavage.

Our results of manipulation indicate that I-r symmetry is not yet established before the third cleavage. Difference between the sinistral and dextral snails is distinguishable as early as at the second or even at the first cleavages (Meshcheryakov, 1975). However, when we altered, by manipulation, the directions of blastomere rotations at the first or at the second cleavage to produce reversed blastomere configuration at the 4-cell stage, the manipulated embryos all developed to perform original-sense third cleavage and resulted in normal 8-cell stage in both the sinistral and the dextral L. stagnalis embryos (Kuroda et al., 2009). We also observed that sinistral embryos occasionally showed dextral-type blastomere arrangement at the 4-cell stage even inside the egg capsules, 
but they all showed normal counterclockwise cleavage at the third division (Kuroda et al., 2009). These results support our conclusion that characteristic macromere - micromere cell contact at the 8-cell stage embryos is the first determining step for asymmetric development of snails, although chirality is indicated before then. The single handedness-determining gene dictates SD and SI at the third cleavage. This agrees well with our previous finding that the handedness- determining gene locus is genetically closely linked to SD and SI, using the congenic F4 (Shibazaki et al., 2004) and F7 (Kuroda et al., 2009) strains as well as F10 strains (Kuroda, 2014) of L. stagnalis we constructed.

The genetically determined critical third cleavage step was overridden by the mechanical micromanipulation, but once chirality is established at this stage, the following development proceeded normally except for the handedness. Body handedness of offspring which were born from the situs inversus mother reverted to the original genetically-determined chirality.

Still we do not know why and how the spiral cleavages occur in alternating manner. It is plausible that this is the way to transfer chirality information from the third cleavage (which is dictated by the single handedness-determining gene locus) to the later developmental stage. In the fourth cleavage, after the recurrent blastomere compaction during post-mitotic phase in which the morphological chirality of embryos was seemingly lost, both the artificially reversed and control embryos exhibited rotation in the opposite sense to the previous third cleavage. Thus, spindle orientation at spiral cleavage stages is controlled by spatial arrangement of blastomeres that is determined by the previous cell cleavage event. How the rotation, especially alternating rotation is achieved is a fascinating topic of research.

\section{Nodal-Pitx pathway operates in P. acuta}

Nodal-Pitx pathway is critically important in the determination of the asymmetric body plan in deuterostomes (Grande and Patel, 2009). The Nodal pathway was not found in Ecdysozoa such as flies and nematodes, but Grande and Patel for the first time showed the asymmetric expression of nodal and its target Pitx genes in the sinistral snail Biomphalaria glabrata and the dextral snail Lottia gigantea (Grande and Patel, 2009). We ourselves reported that recessive sinistral and dominant dextral snails of the same species, L. stagnalis, express nodal and Pitx genes in mirror image patterns (Kuroda et al., 2009). We have shown that when chirality at the 8-cell stage of $P$. acuta embryos was reversed by micromanipulation, the nodal and Pitx expression patterns at the late trochophore stage were completely reversed (Fig. 6), as in the case of $L$. stagnalis.

Nodalis a member of the transforming growth factor- $\beta$ superfamily (Kingsley, 1994; Hogan, 1994). All members of the nodalsubfamily of the TGF- $\beta$ superfamily are synthesized as large prepro precursor molecules, which are cleaved at an RXXR site to release a $C$ terminal peptide of 110-140 amino acids. Nodal orthologue of $P$. acuta ( $P$ aNodal) has RKKR cleavage sequence and the amino acid sequence of the mature domain, particularly after the first of seven cysteine residues is well conserved with that of other members of Lophotrochozoa as well as zebrafish (Ndr2) and Xenopus (Xnr1) (Fig. 5A). This suggests that $P$. acuta's nodal orthologue is also released from the cell and act as a morphogen. Pitx, the target of Nodal, is a homeobox gene that directs the formation of many body structures during early embryonic development (Hamada et al., 2002). Pitx orthologue of $P$. acuta (PaPitx) has a highly conserved homeobox region with mouse, ascidian, sea urchin and other gastropods (Fig. 5B) (Gage and Camper, 1997).

These results may suggest that similar Nodal-Pitx pathway to vertebrates operates in P. acuta as well. In fact, similar Nodal pathway has been found in sea urchins (Duboc and Lepage, 2008; Bessodes et al., 2012). Nodal acts over a distance to elicit dose-dependent responses, and the pathway is tightly regulated by diverse mechanisms including the control of ligand processing, utilization of co-receptors, expression of soluble antagonists, Lefty, as well as positive- and negative-feedback activities (Hamada et al., 2002; Shen, 2007; Müller et al., 2012). Lefty orthologue was reported not found in snails (Grande and Patel, 2009a) and if this were the case, different feedback mechanisms must operate to inhibit the diffusion of Nodal to the other side of midline.

In vertebrates, asymmetric nodal expression occurs in the left side of the lateral plate mesoderm for a short period of time. In contrast, snails express noda/for a longer period of time asymmetrically starting from the 33-64 cell stage (Grande and Patel, 2009a; Kuroda et al., 2009; Kuroda, 2014). Thus, it is quite interesting to find out how the role and regulation mechanisms have changed during the course of evolution. We plan to carry out detailed analysis on nodal expression patterns during the course of development and the contribution toward the chiromorphogenesis using snails which provide simpler model system for study. The mechanisms by which graded signals are generated and interpreted to organismal chiromorphology are of particular interest because they are fundamental for embryonic tissue patterning.

\section{Snails are ideal model animals for the study of I-r symmetry breaking}

Nodal and Pitx genes are not yet expressed by the end of spiral cleavage (fifth cleavage to form from 16 to 24 cell stage), and started to be expressed at 33-64 cell stage (Kuroda, 2014). Here, nodal is already expressed asymmetrically. Thus, spiral cleavage's role may be to transfer chirality information from the third to the fifth cleavage, and then the transferred information at the 24-cell stage is used for the asymmetric expression of nodal. The mechanisms for the spiral cleavages as well as those for leading to the asymmetric nodal gene expression seem to be common for $L$. stagnalis and $P$. acuta, and further, these may be the fundamental mechanisms for the I-r determination in spiralia.

We have shown that the blastomere geometry at the 8-cell stage as a result of the third cleavage is the first step for the handedness determination. It is intriguing to think if the handedness determining gene is the same or similar for $P$. acuta and $L$. stagnalis, how it can act in a completely mirror-imaged manner. There may be some other unknown factors which determine the direction of third cleavage rotation. To find answers to these questions, comparative work on $P$. acuta with $L$. stagnalis or with other members of spiralia is underway.

In the case of $L$. stagnalis, I-r asymmetry is determined already only 3 hours after the first blastomere cleavage, which is determined by a maternal factor before the initiation of zygotic gene expression (Morrill, 1982; Meshcheryakov, 1990). Nodal is expressed asymmetrically at the 33-64 cell stage, about 12 hours after the first cleavage (Kuroda et al., 2009; Kuroda, 2014). These events occur much later in the vertebrates. For example, in the case of mouse, nodal flow and asymmetric nodalexpression were observed 
at E7.5 and E8.25 days, respectively, which means that it is a long step from the first symmetry breaking to the nodal flow/asymmetric nodal expression, which involves many cells and molecules. In contrast, snails' body handedness is determined during the early development period where only a relatively small numbers of cells and molecules are acting. Thus, snails present an excellent model system for studying I-r symmetry breaking.

In conclusion, we have shown a handedness determining pathway starting from the single handedness-determining gene to chiral blastomere arrangement at the 8-cell stage, which then leads to the asymmetric expression of the zygotic genes relevant to asymmetric body formation. During the early developmental stage, consecutive spiral cleavages have important roles, establishing chirality at the spiral third cleavage, and transferring chirality information via spiral fourth and fifth cleavages. That process seems common in sinistral-dominant and dextral-dominant snail species but it leaves questions about the mechanism by which opposite rotation direction is determined. Further studies are required towards an understanding of the symmetry-breaking event in snails.

\section{Materials and Methods}

\section{Snails}

Adult freshwater snails of $P$. acuta were collected at a pond in the Risoukai Nature Park of Tokyo University of Science, or rice field in Noda, Chiba prefecture, Japan. The snails were reared at $25^{\circ} \mathrm{C}$ and given artificial food for tropical fish. In our laboratory condition, the snails laid eggs almost every day.

\section{Micromanipulation}

Manipulation of $P$. acuta embryos at third cleavage was performed as described previously for $L$. stagnalis embryos (Kuroda et al., 2009). Manipulated and non-manipulated embryos were enclosed in a glass capillary tube together with the natural capsular fluid, and cultured for about 2 weeks until they developed into juvenile snails. Time lapse images for the fourth cleavage of manipulated and non-manipulated embryos were obtained using a Zeiss Axioskop2 microscope equipped with an Axiocam MRc colour digital camera, using AxioVision 3.1 software.

\section{Identification of PaNodal and PaPitx and their sequence alignment}

Degenerate primers for isolation of partial sequences of $P$. acuta nodalrelated gene (PaNodal) and Pitx-related gene (PaPitx) were designed from a comparison of nodal and Pitx related proteins in other snails: nodal deg-Fw1 primer, 5'-ATHGGNTGGGGNCARTGGAT-3', corresponding to the amino acid sequence IGWGQWI; nodal deg-Rv1 primer, 5'-GCDATCATNTCNTCRTGRTG-3', corresponding to the amino acid sequence $\mathrm{HH}(\mathrm{D} / \mathrm{E})(\mathrm{D} / \mathrm{E}) \mathrm{MIA}$; Pitx deg-Fw2 primer, 5'-TAYCCNGAYATGGCNACNMG-3', corresponding to the amino acid sequence YPDMATR; and Pitx deg-Rv1 primer, 5'-TTCATRTTNARNCCCCANGGRAA-3', corresponding to the amino acid sequence FPWGLNMN. RT-PCR was performed using cDNA template prepared from the trochophore stage embryos, and amplified PCR fragments were subcloned and identified as nodal or Pitx orthologue partial sequences. The full-length sequences were obtained by 5' and 3' rapid amplification of cDNA ends (RACE) using a SMART ${ }^{\text {TM }}$ RACE CDNA Amplification Kit (Clontech). The GenBank accession numbers for the PaNodaland PaPitx sequences are KJ142140 and KJ142141. The amino acids sequences were aligned with the corresponding genes of deuterostomes and other snails using Clustal X (Jeanmougin et al., 1998). GenBank accession numbers of the nodal-related proteins: mouse Nodal (X70514); Xenopus Xnr-1 (U29447); zebrafish Ndr-2 (AF002219); amphioxus, AmphiNodal (AY083838); ascidian HrNodal (AB069969); sea urchin P.INodal (AY442295); and other gastropods, L. gigantea (EU394708), B. glabrata (EU394707) and L. stagnalis (GU073383). For the Pitx-related proteins: mouse Pitx2 (U80010); amphioxus (AF195616); ascidian (AJ438769); sea urchin (AY714498); and other snails, L. gigantea (EU797117), B. glabrata (EU797116) and L. stagnalis (GU073384).

\section{Whole mount in situ hybridization and immunohistochemistry}

Embryos developed to the late trochophore stage in capillary were fixed in $4 \%$ paraformaldehyde in MTSTr buffer overnight at $4^{\circ} \mathrm{C}$. Digoxigeninlabeled RNA probes were synthesized from the coding regions sequences of cDNA in both antisense and sense strands. Whole mount in situ hybridization was performed as described previously (Kuroda et al., 2009). Stained embryo images were acquired using a Zeiss Axioskop2 microscope. Immunohistochemistry was carried out as described previously (Shibazaki et al., 2004). Images were obtained using a Zeiss LSM5 pascal conforcal microscope with a $20 \times$ objective lens.

\section{Acknowledgements}

We thank JSPS (Japan Society for Promotion of Science) for the financial support (Grant-in-Aid for challenging Exploratory Research: 24657149 to R.K.).

\section{References}

ADAMS D S, ROBINSON K R, FUKUMOTO T, YUAN S, ALBERTSON R C, YELICK $\mathrm{P}, \mathrm{KUO} \mathrm{L}, \mathrm{MCSWEENEY} \mathrm{M}$ and LEVIN M (2002). Early, $\mathrm{H}^{+}-\mathrm{V}$-ATPase-dependent proton flux is necessary for consistent left-right patterning of non-mammalian vertebrates. Development 133: 1657-1671.

BERGMANN D C, LEE M, ROBERTSON B, TSOU M F, ROSE L S and WOOD W B (2003). Embryonic handedness choice in C. elegans involves the Galpha protein GPA-16. Development 130: 5731-5740.

BESSODES N, HAILLOT E, DUBOC V, RÖTTINGER E, LAHAYE F and LEPAGE T (2012). Reciprocal signaling between the ectoderm and a mesendodermal leftright organizer directs left-right determination in the sea urchin embryo. PLoS Genet 8: e1003121.

BLUM M, BEYER T, WEBER T, VICK P, ANDRE P, BITZER E and SCHWEICKERT A (2009). Xenopus, an ideal model system to study vertebrate left-right asymmetry. Dev Dyn 238: 1215-1225.

BOORMAN C J and SHIMELD S M (2002). The evolution of left-right asymmetry in chordates. Bioessays 24: 1004-1011.

CRAMPTON H E (1894). Reversal of cleavage in a sinistral gastropod. Ann NY Acad Sci 8: 167-170.

DUBOC V and LEPAGE T (2008). A conserved role for the Nodal signaling pathway in the establishment of dorso-ventral and left-right axes in deuterostomes. J Exp Zool B Mol Dev Evol 310: 41-53.

GAGE P J and CAMPER S A (1997). Pituitary homeobox 2, a novel member of the bicoid-related family of homeobox genes, is a potential regulator of anterior structure formation. Hum Mol Genet 6: 457-464.

GRANDE C and PATEL N H (2009a). Nodal signalling is involved in left-right asymmetry in snails. Nature 457: 1007-1011.

GRANDE C and PATEL N H (2009b). Lophotrochozoa get into the game: the Nodal pathway and left/right asymmetry in bilateria. Cold Spring Harb Symp Quant Biol 74: 281-287.

HAMADA H, MENO C, WATANABE D and SAIJOH Y (2002). Establishment of vertebrate left-right asymmetry. Nat Rev Genet 3: 103-113.

HOGAN B L, BLESSING M, WINNIER G E, SUZUKI N and JONES C M (1994). Growth factors in development: the role of TGF-beta related polypeptide signalling molecules in embryogenesis. Development Suppl: 53-60.

HOSOIRI Y, HARADA Y and KURODAR (2003). Construction of a backcross progeny collection of dextral and sinistral individuals of a freshwater gastropod, Lymnaea stagnalis. Dev Genes Evol 213: 193-198.

HOZUMI S, MAEDA R, TANIGUCHI K, KANAI M, SHIRAKABE S, SASAMURA T, SPÉDER P, NOSELLI S, AIGAKI T, MURAKAMI R and MATSUNO K (2006). An unconventional myosin in Drosophila reverses the default handedness in visceral organs. Nature 440: 798-802.

JEANMOUGIN F, THOMPSON J D, GOUY M, HIGGINS D G and GIBSON T J (1998). Multiple sequence alignment with Clustal X. Trends Biochem Sci 23: 403-405. 
KINGSLEY D M (1994). The TGF-beta superfamily: new members, new receptors, and new genetic tests of function in different organisms. Genes Dev 8: 133-146.

KURODAR, ENDO B, ABE M and SHIMIZU M (2009). Chiral blastomere arrangement dictates zygotic left-right asymmetry pathway in snails. Nature 462: 790-794.

JURODA R (2014). How a single gene twists a snail. Integr Comp Biol 54: 677-687.

LAMBERT J D (2010). Developmental patterns in spiralian embryos. Curr Biol 20: R72-R77.

LEVIN M, THORLIN T, ROBINSON K R, NOGI T and MERCOLA M (2002). Asymmetries in $\mathrm{H}^{+} / \mathrm{K}^{+}$-ATPase and cell membrane potentials comprise a very early step in left-right patterning. Cell 111: 77-89.

LIU M M, DAVEY J W, BANERJEE R, HAN J, YANG F, ABOOBAKER A, BLAXTER $M L$ and DAVISON A (2013). Fine Mapping of the Pond Snail Left-Right Asymmetry (Chirality) Locus Using RAD-Seq and Fibre-FISH. PLoS One. 8: e71067.

MESHCHERYAKOV V N and BELOUSSOV L V (1975). Asymmetrical Rotations of Blastomeres in Early Cleavage of Gastropoda. Wilhelm Roux's Archives 177: 193-203.

MESHCHERYAKOV V N (1990). The common pond snail Lymnaea stagnalis. In Animal species for developmental studies, Volume 1. Invertebrates (Eds T.A. Dettlaff and S.G. Vassetzky). Consultants Bureau, New York, pp. 69-132.

MORRILL J B (1982). Development of the pulmonate gastropod, Lymnaea. In Developmental biology of freshwater invertebrates (Eds F.W. Harrison and R.R. Cowden). Alan R. Liss, New York, pp. 399-483.

MÜLLER P, ROGERS K W, JORDAN B M, LEE J S, ROBSON D, RAMANATHAN $S$ and SCHIER A F (2012). Differential diffusivity of Nodal and Lefty underlies a reaction-diffusion patterning system. Science 336: 721-724.

NONAKA S, TANAKA Y, OKADA Y, TAKEDA S, HARADA A, KANAI Y, KIDO M and
HIROKAWA N (1998). Randomization of left-right asymmetry due to loss of noda cilia generating leftward flow of extraembryonic fluid in mice lacking KIF3B motor protein. Cell 95: 829-837.

NONAKAS, SHIRATORI H, SAIJOH Y and HAMADAH (2002). Determination of leftright patterning of the mouse embryo by artificial nodal flow. Nature 418: 96-99.

PELSENEERP (1920). Les variations et leur hérédité chez les mollusques. Memoires Acad Royale de Belgique Deuxième série 5: 1-826.

SCHILTHUIZEN M and DAVISONA (2005). The convoluted evolution of snail chirality. Naturwissenschaften 92: 504-515.

SHEN M M (2007). Nodal signaling: developmental roles and regulation. Development 134: 1023-1034.

SHIBAZAKI Y, SHIMIZU M and KURODA R (2004). Body handedness is directed by genetically determined cytoskeletal dynamics in the early embryo. Curr Biol 14: 1462-1467.

SPÉDERP, ADÁM G and NOSELLIS (2006). Type ID unconventional myosin controls left-right asymmetry in Drosophila. Nature 440: 803-807.

SPÉDER P, PETZOLDT A, SUZANNE M and NOSELLI S (2007). Strategies to establish left/right asymmetry in vertebrates and invertebrates. Curr Opin Gene Dev 17: 351-358.

VANDENBERG L N and LEVIN M (2009). Perspectives and open problems in the early phases of left-right patterning. Semin Cell Dev Biol 20: 456-463.

VERDONK N H and VAN DEN BIGGELAAR J A M (1983). Early development and the formation of the germ layers. In The Mollusca, Volume 3. Development (Eds N.H. Verdonk, J.A.M. van den Biggelaar and A.S. Tompa). Academic Press, New York, pp. 91-122.

WILLIAMS J M (1887). A dextral Physa fontinalis. J. Conchology 5: 220. 


\section{Further Related Reading, published previously in the Int. J. Dev. Biol.}

Brachyury, Tbx2/3 and sall expression during embryogenesis of the indirectly developing polychaete Hydroides elegans Cesar Arenas-Mena

Int. J. Dev. Biol. (2013) 57: 73-83

http://dx.doi.org/10.1387/ijdb.120056ca

Planarian embryology in the era of comparative developmental biology Jose M. Martin-Duran, Francisco Monjo and Rafael Romero Int. J. Dev. Biol. 56: 39-48 (2012)

doi: $10.1387 / \mathrm{ijdb} .113442 \mathrm{jm}$

Heart formation and left-right asymmetry in separated right and left embryos of a newt Kazuhiro Takano, Yuzuru Ito, Shuichi Obata, Tsutomu Oinuma, Shinji Komazaki, Hiroaki Nakamura and Makoto Asashima

Int. J. Dev. Biol. 51: 265 - 272 (2007)

doi: $10.1387 / \mathrm{ijdb} .072270 \mathrm{kt}$

The dynamic nature of mollusc egg surface architecture and its relation to the microtubule network

Sheena E.B. Tyler and Susan J. Kimber

Int. J. Dev. Biol. 50: 405 - 412 (2006)

doi: $10.1387 / \mathrm{ijdb} .052007 \mathrm{st}$

Xenopus nodal related-1 is indispensable only for left-right axis determination Ryuji Toyoizumi, Tsuyoshi Ogasawara1, Shigeo Takeuchi and Kazue Mogi Int. J. Dev. Biol. 49: 923 - 938 (2005) doi: $10.1387 / \mathrm{ijdb} .052008 \mathrm{rt}$

Xenopus neurula left-right asymmetry is respeficied by microinjecting TGF-beta5 protein.

Kazue Mogi, Madoka Goto, Eri Ohno, Yoshitaka Azumi, Shigeo Takeuchi and Ryuji Toyoizumi Int. J. Dev. Biol. 47: 15 - 29 (2003)

5 yr ISI Impact Factor $(2011)=2.959$

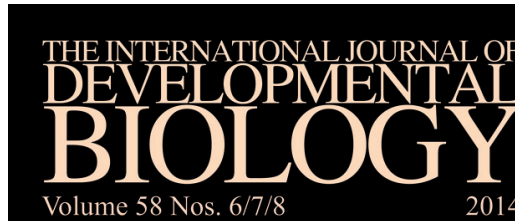

Volume 58 Nos. 6/7/8 2014

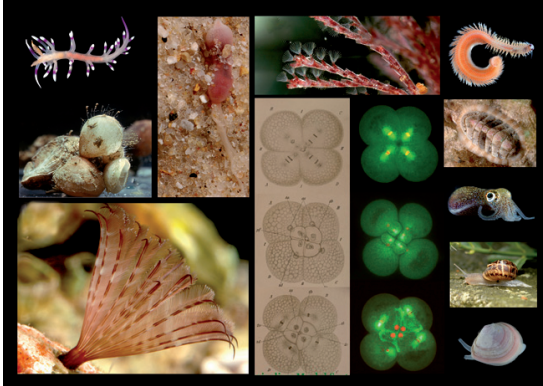

Spiralian Model Systems

ק.

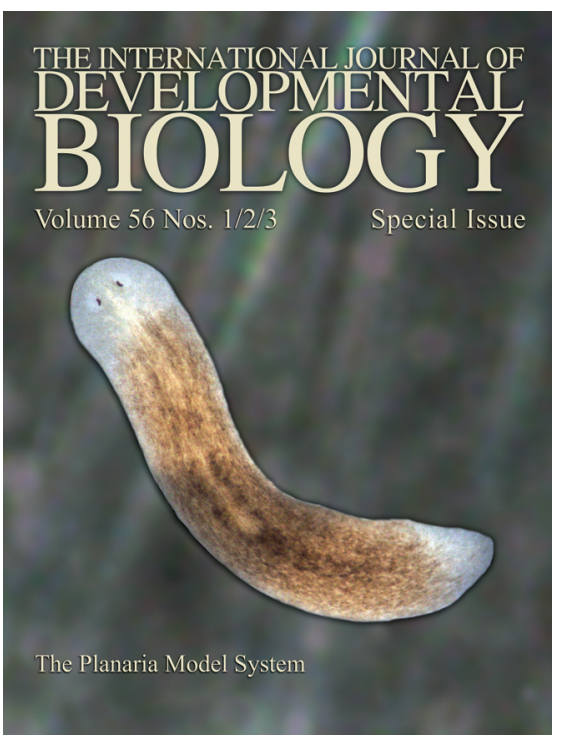

